\title{
Quantifying NMR relaxation correlation and exchange in articular cartilage with time domain analysis
}

\section{Authors: Sarah E. Mailhiot, Fangrong Zong, James E. Maneval, Petrik Galvosas, Joseph D. Seymour.}

NOTICE: this is the author's version of a work that was accepted for publication in Journal of Magnetic Resonance Changes resulting from the publishing process, such as peer review, editing, corrections, structural formatting, and other quality control mechanisms may not be reflected in this document. Changes may have been made to this work since it was submitted for publication. A definitive version was subsequently published in Journal of Magnetic Resonance, [V287, February 2018]. DOI\#10.1016/j.jmr.2017.12.014

Mailhiot SE, F Zong, JE Maneval, RK June, P Galvosas, JD Seymour, "Quantifying NMR relaxation correlation and exchange in articular cartilage with time domain analysis," Journal of Magnetic Resonance. February 2018; 287:82-90. 


\title{
Quantifying NMR relaxation correlation and exchange in articular cartilage with time domain analysis
}

\author{
Sarah E. Mailhiot, Fangrong Zong, James E. Maneval, Ronald K. June, Petrik \\ Galvosas, Joseph D. Seymour
}

\begin{abstract}
Measured nuclear magnetic resonance (NMR) transverse relaxation data in articular cartilage has been shown to be multi-exponential and correlated to the health of the tissue. The observed relaxation rates are dependent on experimental parameters such as solvent, data acquisition methods, data analysis methods, and alignment to the magnetic field. In this study, we show that diffusive exchange occurs in porcine articular cartilage and impacts the observed relaxation rates in $\mathrm{T}_{1}-\mathrm{T}_{2}$ correlation experiments. By using time domain analysis of $\mathrm{T}_{2}-\mathrm{T}_{2}$ exchange spectroscopy, the diffusive exchange time can be quan-tified by measurements that use a single mixing time. Measured characteristic times for exchange are commensurate with $T_{1}$ in this material and so impacts the observed $\mathrm{T}_{1}$ behavior. The approach used here allows for reliable quantification of NMR relaxation behavior in cartilage in the presence of diffusive fluid exchange between two environments.
\end{abstract}

Cartilage has been widely studied by NMR relaxation methods $[1-10] . \mathrm{T}_{2}$ relaxation has been correlated to the health of the tissue with increases in observed $T_{2}$ values occurring when cartilage is damaged [5]. Though $\mathrm{T}_{2}$ weighted magnetic resonance imaging (MRI) has been used to track disease caused degradation of carti-lage such as arthritis, the standard for clinical diagnosis of arthritis in the USA is still X-ray imaging and not MRI.

One aspect which limits MRI methods in this regard is the wide variability in reported $\mathrm{T}_{2}$ relaxation rates across studies [11] and the difficulty in connecting the observation of multiple sites, pop-ulations of different rotational and molecular mobility, to disease progression and diagnosis. Relaxation in cartilage has been shown to be composed of multiple relaxation rates depending on experi-mental conditions. Observation of multicomponent $T_{2}$ in articular cartilage depends on several experimental parameters and results from imaging experiments indicate from one to five distinct $T_{2}$ relaxation rates $[1,2,4,11-14]$.
Exchange of material between sites can also lead to observation of multiple relaxation rates in cartilage [1519]. In healthy human cartilage, relaxation exchange spectroscopy (REXSY) measured two site exchange at a mixing time of $12 \mathrm{~ms}$ [20]. Exchange between protons experiencing the magic angle effect, i.e. an increase in $T_{2}$ of fibrils and associated water aligned at $54.7 \square$ to $B_{0}$, and protons in the bulk pore fluid was measured in canine cartilage soaked in phosphate buffered saline (PBS) [12]. These studies did not deter-mine an exchange rate in cartilage but demonstrated that the exchange rate impacts the observable relaxation in cartilage, and that the time scale of exchange can be probed with NMR. Hence the value and number of observed relaxation rates measured must be interpreted in the context of exchange processes to quantify the effect of exchange on the intrinsic relaxation rates. A method to do so is presented here.

A consideration in the use of NMR relaxation methods in carti-lage, and biological materials in general, is that such methods involve long experiment times. For example, the $\mathrm{T}_{2}-\mathrm{T}_{2}$ REXSY experiment is composed of two Carr-Purrcell-Meiboom-Gill (CPMG) pulse trains separated by a mixing or storage time in which spins are stored in the longitudinal axis [15] [Fig. 1a]. The duration of these experiments can take from $30 \mathrm{~min}$ to several hours depending on signal-to-noise considerations and phase 


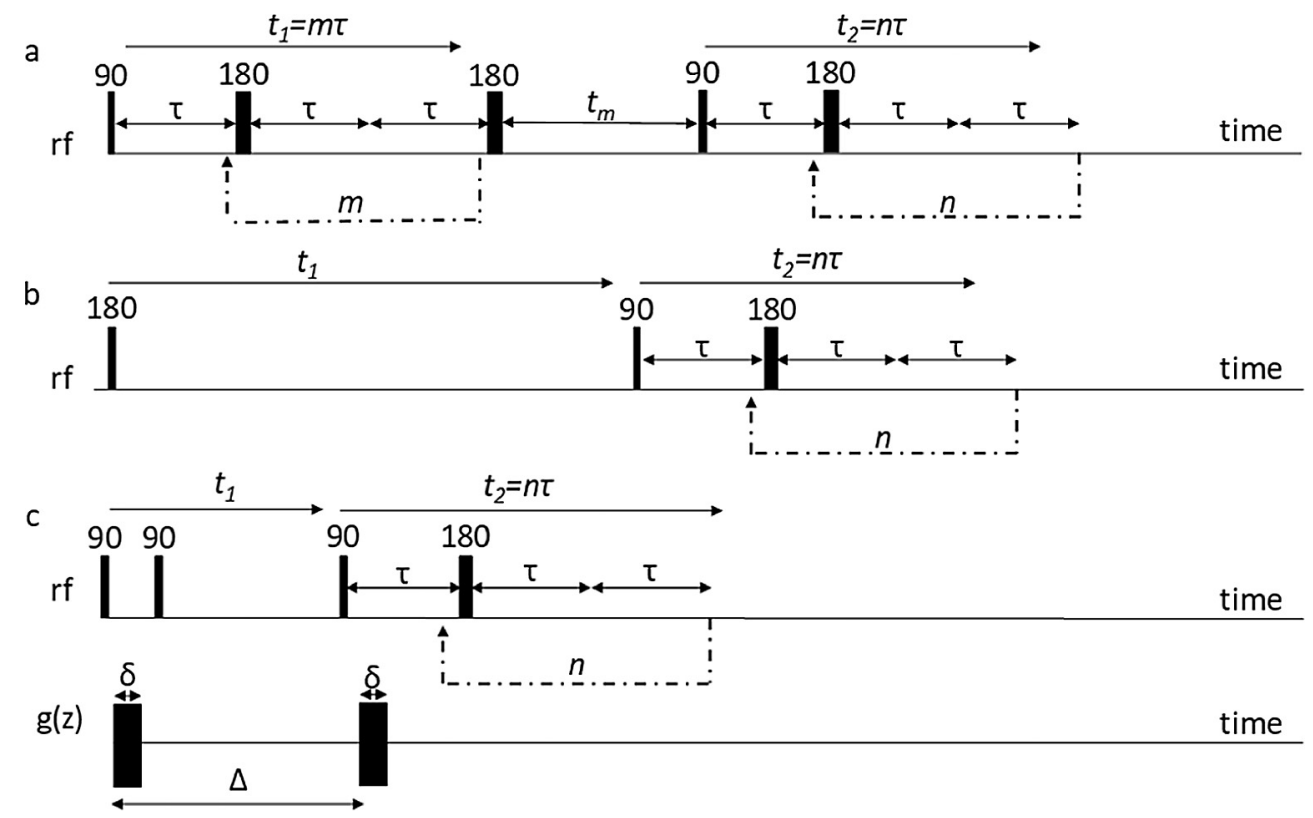

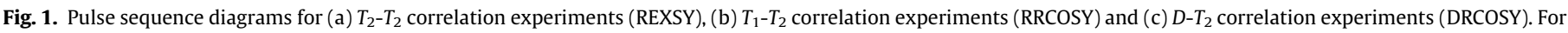

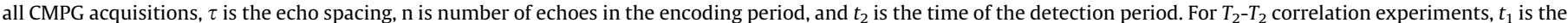

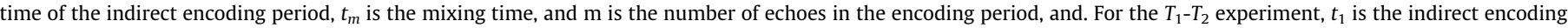

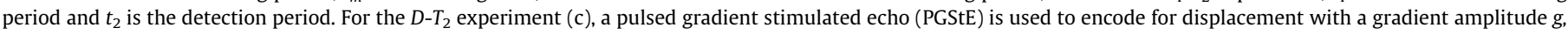
duration $\delta$, and observation time $\Delta$.

cycles needed to provide useable data. The usual practice is to repeat measurements for a range of mixing times leading to three-dimensional datasets and long experiment times [15,21]. In contrast to samples from petrophysical sources and engineered materials, biological samples are subject to degradation with time ex vivo and long-duration experiments for in vivo measurements are difficult, if not impossible. Therefore, it is critical to reduce experiment time in multi-dimensional relaxation methods by better understanding of the physical processes in a material. Recent improvements in sampling strategies $[22,23]$ have accelerated data acquisition. Although methods using spatially resolved acquisition [23] are not applicable to spatially heterogeneous samples as studied here, the development of sampling strategies can benefit from the approach presented here.

The analysis and use of multi-dimensional NMR measurements of relaxation and diffusion provides clear evidence of two distinct sites in porcine cartilage samples randomly oriented with respect to the magnetic field. These results demonstrate that exchange of water occurs between physically different relaxation sites on the time scale of seconds. By applying analytical relaxation-exchange models, we show how the exchange between these cartilagecomponent sites impact measured relaxation rates and that taking such impacts into account enhances the understanding of NMR relaxation measurements in cartilage. These results could lead to improvements and extension in their use in research and correlation with clinical settings.

\section{Background and theory}

Magnetization relaxation in cartilage has been shown to be multi-exponential with a dependence on a range of experimental factors. The accurate measurement of $T_{2}$ in cartilage is dependent on factors including data analysis methods, data acquisition methods, and sample preparation [2-6,8-11,13,14,24-28]. One factor that is not well quantified in articular cartilage is exchange of water amongst the various structures and regions in a sample. Previous studies have shown that exchange in cartilage alters observed relaxivities, as opposed to intrinsic local relaxivities [12] and reduces the number of distinct observed relaxation times due to exchange averaging.

As noted, the usual aim of measuring relaxation processes in cartilage is to associate specific relaxation times with specific anatomical features of the cartilage. To accomplish this aim, studies need to be clear about how both the number of sites observed and the values of the NMR parameters that characterize the sites are determined. Multi-dimensional relaxation-time measurements in cartilage and other structured materials can then be used to answer two important questions about the material. First, are there distinct sites in a material and, if so, how many are there? If multiple distinct sites are observed for a sample, the next question is then what are the values of the NMR parameters that distinguish them?

The assessment of sites and their number is typically answered by assessing whether or not there is more than one distinct mode or peak in a relaxation-time spectrum. Since NMR relaxation processes are often first-order processes, the key tool here is the inverse Laplace transform (ILT) that converts the time-domain data from an experiment into a suitable relaxation-time spectrum $[29,30]$. The modes in these spectra are identified by the relaxation time along with an amplitude or fraction. Though there is still discussion as to the exact meaning of the peaks in an ILT of a relaxation experiment [16], a properly computed ILT provides a powerful tool for assessing NMR relaxation in a sample.

If there are multiple peaks in a relaxation spectrum, the question of what parameters best characterize the sites that give rise to the peaks is often addressed by extracting NMR parameters through data-fitting. Though the peaks in an ILT can be used for this purpose $[18,21]$, the approach taken in this study is to use the methods of time-domain analysis [31]. These methods proceed by proposing a model of the underlying exchange processes in a sample and then adjust the parameters of that model to achieve the best fit to the data.

Time domain analysis of multi-dimensional relaxation experiments [16-18,31,32] such as the single-mixing-time REXSY has 
been shown to effectively quantify the exchange rates between sites in borosilicate and soda-lime glass bead packs [33], cement [18], urea samples [17], and a silica bead pack [16]. In this work, the goal is to extend this method to cartilage and quantify the exchange phenomena between sites.

The time-domain model that will be used here is a two-site specialization of the multi-site relaxation model that was first introduced by McConnell in 1958 [34]. Briefly, this model can be developed from a magnetization balance and is expressed as,

$d / d t\left[\begin{array}{l}M_{a}-M_{a}^{e q} \\ M_{b}-M_{b}^{e q}\end{array}\right]=\left[\begin{array}{cc}-R_{a}-k_{a} & k_{b} \\ k_{a} & -R_{b}-k_{b}\end{array}\right]\left[\begin{array}{l}M_{a}-M_{a}^{e q} \\ M_{b}-M_{b}^{e q}\end{array}\right]$

where $R_{a}$ and $R_{b}$ are the intrinsic relaxation rates either longitudinal or transverse for the two sites ( $a$ and $b$ ), and $k_{a}$ and $k_{b}$ are the exchange rate coefficients that characterize transfer from site $a$ to $b$ and site $b$ to $a$, respectively. In these expressions, $M_{a}$ and $M_{b}$ are the site magnetizations with $M_{a}^{e q}$ and $M_{b}^{e q}$ providing the site magnetization values that are in thermal equilibrium with the applied field. The balance in Eq. (1) can be written in compact form as

$\mathrm{d} \boldsymbol{M} / \mathrm{dt}=\boldsymbol{A} \boldsymbol{M}$

where $\boldsymbol{M}$ is a two-element vector of the site magnetizations and the matrix $\boldsymbol{A}$ holds the combination of relaxation and exchange coefficients as

$\boldsymbol{A}=[\boldsymbol{R}+\boldsymbol{K}]=\left[\begin{array}{cc}-R_{a}-k_{a} & k_{b} \\ k_{a} & -R_{b}-k_{b}\end{array}\right]$

The time-domain approach uses Eq. (2) to model the evolution of the magnetization during inter-pulse portions of a particular pulse sequence with the relaxation terms accounting for transverse or longitudinal processes as appropriate to an interval. Initial conditions for the solution of $\mathrm{Eq} \mathrm{(2)} \mathrm{for} \mathrm{each} \mathrm{interval} \mathrm{in} \mathrm{a} \mathrm{pulse}$ sequence is provided by the rf pulses preceding magnetization evolution. Fitting is accomplished by adjusting the parameters in the $\boldsymbol{A}$ matrices for the sequence so that the predictions of the model match the data of the experiment in a least-squares sense $[16,17,31]$.

The analytical solution to Eq. (2) is possible and has been provided for particular pulse sequences discussed here by Van Landeghem et al. and Dortch et al. [16,17]. A useful outcome of these solutions is the ability to predict the amplitudes of the peaks in an ILT, the so-called peak-matrix, $\boldsymbol{P}$ [17].

Expressions for the $\boldsymbol{P}$ matrices for the pulse sequences discussed in this paper are

$\boldsymbol{P}_{\boldsymbol{T}_{2} \boldsymbol{T}_{2}}=2\left[\boldsymbol{U}_{2}^{-1} \exp \left(\left[\boldsymbol{R}_{1}+\boldsymbol{K}\right] t_{m}\right) \boldsymbol{U}_{2}\right]^{\circ}\left[\boldsymbol{U}_{2}^{-1} \boldsymbol{M}(0) \mathbf{1}_{1 \boldsymbol{x} N} \boldsymbol{U}_{2}\right]^{T}$

for the $T_{2}-T_{2}$ sequence (Fig. 1a) and

$\boldsymbol{P}_{\boldsymbol{T}_{1} \boldsymbol{T}_{2}}=2\left[\boldsymbol{U}_{2}^{-1} \exp \left(\left[\boldsymbol{R}_{1}+\boldsymbol{K}\right] t_{1}\right) \boldsymbol{M}(0)\right]^{\circ}\left[\mathbf{1}_{1 \boldsymbol{x} N} \boldsymbol{U}_{2}\right]^{\mathrm{T}}$

for the $T_{1}-T_{2}$ sequence (Fig. $1 \mathrm{~b}$ ). In these expressions eigenvectors, $\boldsymbol{U}_{1,2}$, are the matrix of eigenvectors obtained from the matrix $\boldsymbol{R}_{1,2}$

$+\boldsymbol{K}, \boldsymbol{M}(0)$ is the initial magnetization for the sequence, typically the equilibrium magnetization, $t_{1}$ and $t_{m}$ are specified by the timing diagrams (Fig. 1) and $\mathbf{1}_{1 \times N}$ is an $\mathrm{N}$-element row vector of ones. The symbol ${ }^{\circ}$ represents element-wise (or Hadamard) matrix multiplication between two factors. The reader is referred to the reference of Dortch, et al. [17] for further details on these expressions.

Another recently introduced and robust time domain visualization method for $T_{2}-T_{2}$ data is used to confirm diffusive coupling [35]. The signal is described by the function $S\left(t_{1}, t_{2}\right)$ for the timings from Fig. 1a. In a model where spins exchange between sites $a$ and $b$, the signal for symmetric exchange peaks is given by

$S\left(t_{1}, t_{2}\right)=0.5 \exp \left(-t_{1} R_{2 a}-t_{2} R_{2 b}\right)+0.5 \exp \left(-t_{1} R_{2 b}-t_{2} R_{2 a}\right)$.
On diagonal peaks exist at $R_{2 a}$ and $R_{2 b}$. Using principal axes around the symmetric axis of the $T_{2}-T_{2}$ data, relaxation can be defined as $R_{s}=1 / 2\left(R_{2 a}+R_{2 b}\right)$ and $R_{d}=1 / 2\left(R_{2 a}-R_{2 b}\right)$ and symmetry time domain axes $\tau_{s}=t_{1}+t_{2}$ and $\tau_{d}=t_{1}-t_{2}$. By substituting these transformations into Eq. (6), a simplified expression for exchange peaks is derived

$S\left(\tau_{s}, \tau_{d}\right)=\exp \left(-\tau_{s} R_{S}\right) \cosh \left(\tau_{d} R_{d}\right)$.

If exchange occurs $\left(R_{\mathrm{d}} \neq 0\right)$ in a sample, a plot of the acquired signal as a function of $\tau_{\mathrm{d}}$ at fixed $\tau_{\mathrm{s}}$ will show a hyperboliccosine shape [35].

\section{Experimental}

Articular cartilage samples were obtained from the shoulder joint of skeletally mature pigs. For the measurements reported here, cartilage from three distinct individuals was used to assess repeatability and accuracy of the methods. Cartilage samples were cylindrical with $4 \mathrm{~mm}$ diameters and heights that reflect the full thickness of the cartilage, about 0.5 to $1 \mathrm{~mm}$, as it was separated from the bone with a scalpel. The samples were rinsed in phosphate buffered saline (PBS) for $20 \mathrm{~min}$ to remove blood and fat. Each NMR experiment used a $5 \mathrm{~mm}$ glass NMR tube containing ten cartilage cylinders which were randomly oriented within the tube and, hence, with respect to the static field. The fluid in the tubes was only that held by the cartilage samples and excess fluid well below the radio frequency coil. No solvent was present in the inter-cylinder space within the tubes.

Relaxation experiments consisted of traditional onedimensional methods for $T_{1}$ and $T_{2}$ measurement and twodimensional methods. One-dimensional measurements for $T_{2}$ estimation were made using a CPMG sequence [36] with single point acquisition. An echo spacing (TE) of $0.50 \mathrm{~ms}$ was used and the acquisition of 2048 echoes provided an acquisition time of $1.05 \mathrm{~s}$. With a 4-step phase cycle and 4 averages per phase-cycle step, total experiment time was $45 \mathrm{~s}$. Analogous measurements for $T_{1}$ were made with an inversion-recovery sequence. The 180-90 $\mathrm{rf}$ inter-pulse delays were logarithmically spaced from $0.001 \mathrm{~s}$ to $6 \mathrm{~s}$ for 30 values. A recovery delay of $10 \mathrm{~s}$ was used along with 4 step phase cycle and 4 averages for each phase-cycle step. Total experiment time for these $T_{1}$ measurements was $40 \mathrm{~min}$.

Pulse sequence diagrams for the two-dimensional relaxationmeasurement methods are shown in Fig. 1 . The REXSY $\left(T_{2}-T_{2}\right)$ experiment in Fig. 1a [15] consists of a CMPG encode period with $m$ echoes to encode for $T_{2}$, followed by a $T_{1}$ storage period with a mixing time, $t_{m}$, from $5 \mathrm{~ms}$ to $1000 \mathrm{~ms}$ and then single-point measurement of the CPMG echo train comprised of $n=2048$ echoes with an echo spacing $2 \tau$ of $0.50 \mathrm{~ms}$. Pulse-sequence repetition time was $60 \mathrm{~s}$ to eliminate sample heating. A 16-step phase cycle was used with 16 averages per step to give a total experiment time of $4 \mathrm{~h}$. The $T_{1}-T_{2}$ measurement method [30] is given in Fig. 1b. In the first section of the pulse sequence, a $180^{\circ}$ inversion recovery sequence is used with 30 delay times logarithmically spaced from $0.001 \mathrm{~s}$ to $6 \mathrm{~s}$ as in the 1D experiment. After the inversion delay, a single-point CPMG echo train comprised of $n=2048$ echoes with a $0.5 \mathrm{~ms} 180^{\circ}$ pulse spacing. Pulse-sequence repetition time was $10 \mathrm{~s}$. A 4-step phase cycle with 8 averages per phase-cycle step leads to a total experiment time of $25 \mathrm{~min}$.

Diffusion- $T_{2}$ relaxation correlation measurements were made with the $D-T_{2}$ sequence [37,38] (Fig. 1c). The sequence consists of a pulsed gradient stimulated echo (PGStE) sequence that encodes for self-diffusion coupled to the acquisition of a single-point echo train of $n=2048$ echoes with an echo spacing $2 \tau$ of $0.5 \mathrm{~ms}$. For PGStE, a displacement observation time $(\Delta)$ of $50 \mathrm{~ms}$ and a gradient duration time $(\delta)$ of $1.6 \mathrm{~ms}$ were used. Gradient strength, $g$, 
was varied from 0 to $8 \mathrm{~T} \mathrm{~m}^{-1}$ in 32 linearly spaced steps. The pulse sequence repetition time was $10 \mathrm{~s}$ and an 8 -step phase cycle with 16 averages per phase-cycle step led to a total experiment time of $2.5 \mathrm{~h}$.

Measurements were taken on a Bruker 9.4 T vertical wide bore superconducting magnet with an AVANCE spectrometer operating at $400 \mathrm{MHz}$. A Bruker Diff60 with a maximum magnetic field gradient strength in the z-direction of $35 \mathrm{Tm}^{-1}$ at $60 \mathrm{~A}$ was used. A $5 \mathrm{~mm}$ saddle radio frequency coil with $90^{\circ}$ and $180^{\circ}$ pulses of $5 \mu$ s at $50 \mathrm{~W}$ provided signal excitation and detection. Data was collected using TopSpin 2.1 software (Bruker Biospin, Karlsruhe, Germany).

The one and two-dimensional Inverse Laplace Transform (ILT) are used for data analysis of multi-exponential decays [30]. Biexponential expressions are fit to the one dimensional time domain data [39]. The bi-exponential time domain fits are then compared to one-dimensional ILT results. These data are helpful in providing starting points for the data-fitting methods described by Van Landeghem et al. and Dortch et al. [16,17] for finding the parameters in the two site model of Eq. (2).

\section{Results and discussion}

The first goal is to use the measurements to establish if there are distinct relaxation environments in the material and, if so, how many sites there are. Fig. 2a and b show one-dimensional relaxation spectra for transverse and longitudinal relaxation experiments determined by the ILT. The table in Fig. 1c summarizes the biexponential fits in the time domain for the three samples used in the study to provide a mean and standard deviation of the relaxation time values and percent total signal in each population.

There are two peaks in the $T_{2}$ spectrum and the $T_{2}$ fit summary indicates that the values determined from the time domain fit are statistically different across the samples. However, there is only one mode in the $T_{1}$ spectrum (Fig. 1b). Attempts to fit a biexponential model to the $T_{1}$ time domain data does not deliver any statistical support for two distinct $T_{1}$ values. Based on the $T_{2}$ results, we tentatively conclude that there are two distinct sites in the material. Confirmation of this result and interpretation of why there is only one observed $T_{1}$ value will be provided in the discussion of the time-domain fitting results below.

Establishing the existence of two distinct sites with differing mobilities in this material will be carried out by observing differences in the macromolecular environment of water as determined by the $T_{2}$ parameters. A key first step is to ensure that neither population can be interpreted as a free solvent site in terms of the measurement of $T_{1}-T_{2}[19,30,40]$ and $D-T_{2}$ spectra [41]. Sites are physical domains of varying mobility in the material which generate populations of a certain amplitude in the data. These twodimensional experiments provide diffusion and $T_{1}$ trends and values that allow better understanding of the state of the fluid in the cartilage matrix. $T_{1}-T_{2}$ measurements (Fig. 3a) build on the 1D $T_{2}$ spectral results and show two well-defined sites in this cartilage material. The populations are located off the parity line $T_{1}=T_{2}$ where data representative of a free solvent site would appear. The further a population is from the parity line, the more the rotational mobility of the molecules is restricted [30]. The appearance of two sites with the same $T_{1}$ value indicates one of the populations could be an exchange cross peak [17]. The $T_{1}$ and $T_{2}$ values at the center of the populations in Fig. 3a are consistent with the values found with one dimensional experiments (Fig. 2c).

$D-T_{2}$ measurements (Fig. 3b) also show two well-defined sites and confirm the existence of two sites in the material that are restricted in the pore space of the cartilage matrix based on the diffusion amplitude. Though the sites have different $T_{2}$ environments, both sites have essentially the same translational mobility characterized by a diffusion coefficient, $D=1.47 \times 10^{-9} \mathrm{~m}^{2} \mathrm{~s}^{-1}$ and $D=$ $1.35 \times 10^{-9} \mathrm{~m}^{2} \mathrm{~s}^{-1}$, values that are about $75 \%$ that of free water $\left(D_{0}=2.1 \times 10^{-9} \mathrm{~m}^{2} \mathrm{~s}^{-1}\right.$ at $\left.25^{\circ} \mathrm{C}\right)$. Additionally, there is little sample-to-sample variability of the translational diffusion, as seen by the small standard deviation of the diffusion across samples, despite larger percentage variations in the relaxation behavior (Fig. 3c). This indicates that the translational mobility of the fluid in both sites is restricted by interactions with the cartilage matrix and suggests that the measured fluid phases are associated with the cartilage structures and not simply external to the cartilage as free solvent. To reiterate, the $T_{1}-T_{2}$ and $D-T_{2}$ correlations support results from the $1 \mathrm{D} T_{2}$ spectra that there are two distinct mobility a

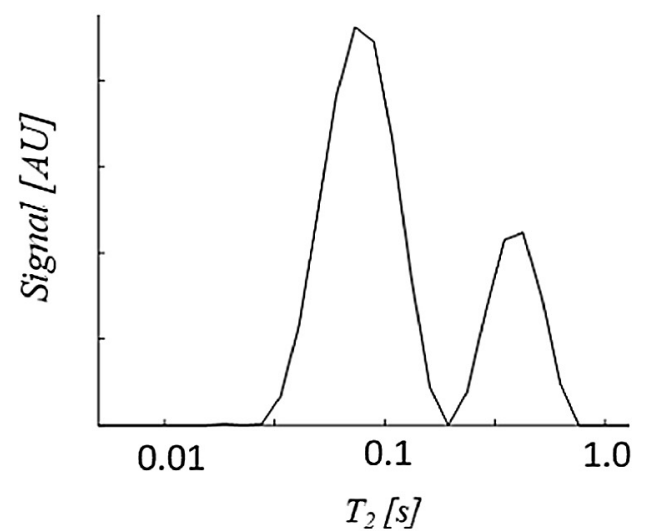

b

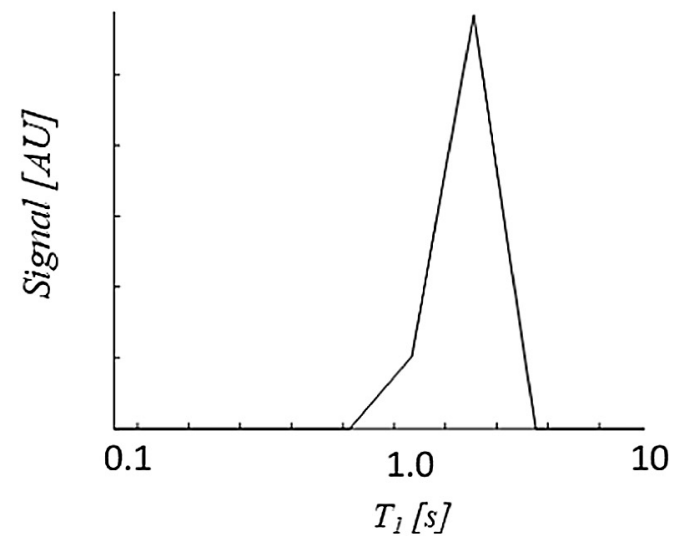

C

\begin{tabular}{|l|l|l|l|l|}
\hline$T_{2 A}[\mathrm{~ms}]$ & $T_{2 B}[\mathrm{~ms}]$ & $T_{1 A, B}[\mathrm{~ms}]$ & $M_{A}[\%]$ & $M_{B}[\%]$ \\
\hline $77 \pm 2$ & $182 \pm 28$ & $2167 \pm 94$ & $36 \pm 9$ & $64 \pm 9$ \\
\hline
\end{tabular}

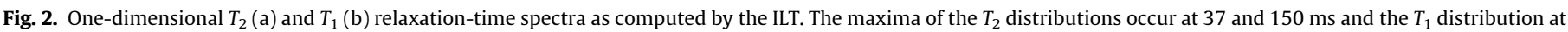

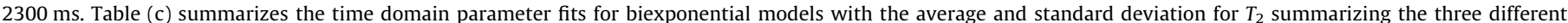

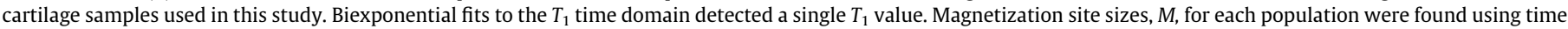
domain bi-exponential fitting of one dimensional CPMG data. 

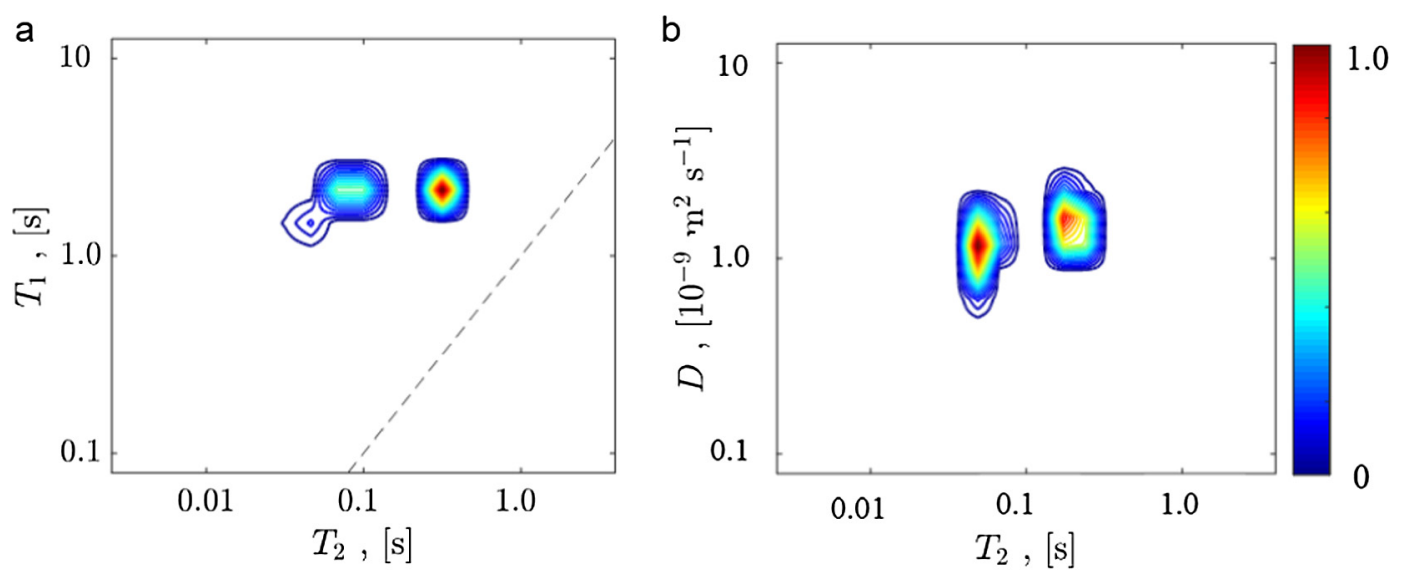

\begin{tabular}{|l|l|l|}
\hline$T_{1} / T_{2 A}$ & $T_{1} / T_{2 B}$ & $D / D_{0}$ \\
\hline $5.39 \pm 2.64$ & $19.2 \pm 10.8$ & $0.74 \pm 0.01$ \\
\hline
\end{tabular}

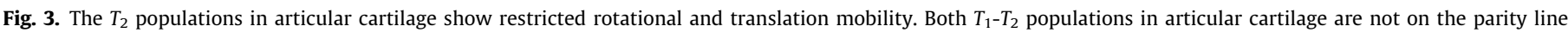

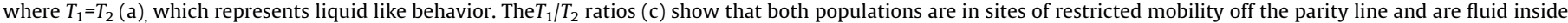

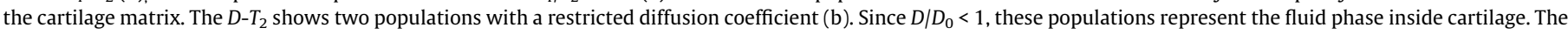
average and standard deviation shown in (c) is taken over three cartilage samples.

environments in these samples. These sites contain fluid that is associated with the cartilage matrix, resulting in similar restricted translational diffusion represented by a common $D$ value and different rotational mobility represented by two distinct $T_{2}$ values.

The $D-T_{2}$ and $T_{1}-T_{2}$ relaxation and diffusion experiments results establish the existence of two distinct sites in a sample. These experiments also provide insights into the mobility and relaxation environments that lead to these sites being unique, in terms of $T_{2}$.

We note from Fig. 3 that exchange between sites would not be identified on the basis of $T_{1}$ and $D$ measurements alone. However, by including $T_{2}$ in the measurement protocol, multiple peaks in the ILT are observed and exchange can be explored in an effective manner via the $T_{2}-T_{2}$ experiment.

The $T_{2}-T_{2}$ experiment has an experimentally controlled mixing time, $t_{m}$. By varying this mixing time, $T_{2}-T_{2}$ measurements can be used to probe exchange processes between sites $[16-18,21,31,32,42]$. In this study, the mixing time was varied between 1 and $1000 \mathrm{~ms}$ as shown in Fig. 4 . At $1 \mathrm{~ms}$, exchange peaks are not visible (Fig. 4a), and by $1000 \mathrm{~ms}$, the exchangepeak signal makes up only about $1.5 \%$ of the total signal (Fig. 4c). The appearance of exchange peaks at $1000 \mathrm{~ms}$ suggests that exchange between the sites is occurring on the time scale of about one second $[16,17]$ and hence a rough estimate of the exchange rate coefficient is $k_{a} \sim 1 \mathrm{~s}^{-1}$. The exchange rate $k_{a}$ is related to $k_{b}$ by mass balance such that $k_{a}=k_{b} \frac{M_{b}}{M_{a}}$. In this work, we will refer to $k_{a}$ as the exchange rate.

We can refine this estimate of $k_{a}$ by fitting the 2-site relaxationexchange model (Eqs. (2) and (3)) tuned specifically for the $T_{2}-T_{2}$ sequence (Fig. 1a). The fitting process not only provides a value for $k_{a}$, but also estimates of the intrinsic $T_{1}, T_{2}$, values for each site as well as the site population percentage, $M$. The results for the three samples in this study using measurements with mixing times of $500 \mathrm{~ms}$ are summarized in Table 1 along with comparable results from the $1 \mathrm{D}$ methods. The $500 \mathrm{~ms}$ mixing time was chosen to reduce $T_{1}$ weighting, thus improving SNR for a more stable time domain fitting, and to show that the presence of detectable exchange cross peaks in the 2D data is not required for this analysis approach to work.

Agreement between the parameter values measured by the one- and two-dimensional approaches is good with the best performance obtained for the $T_{2}$ values. As was the case with the 1D $T_{1}$ data fitting discussion, the $2 \mathrm{D}$ model predicts essentially the same $T_{1}$ value for both sites. This result can be clearly explained by the two-site model as being due to the fact that the timescale for exchange between the two sites (about $1.5 \mathrm{~s}$ ) is approximately that of the time-scale for longitudinal relaxation. The rate
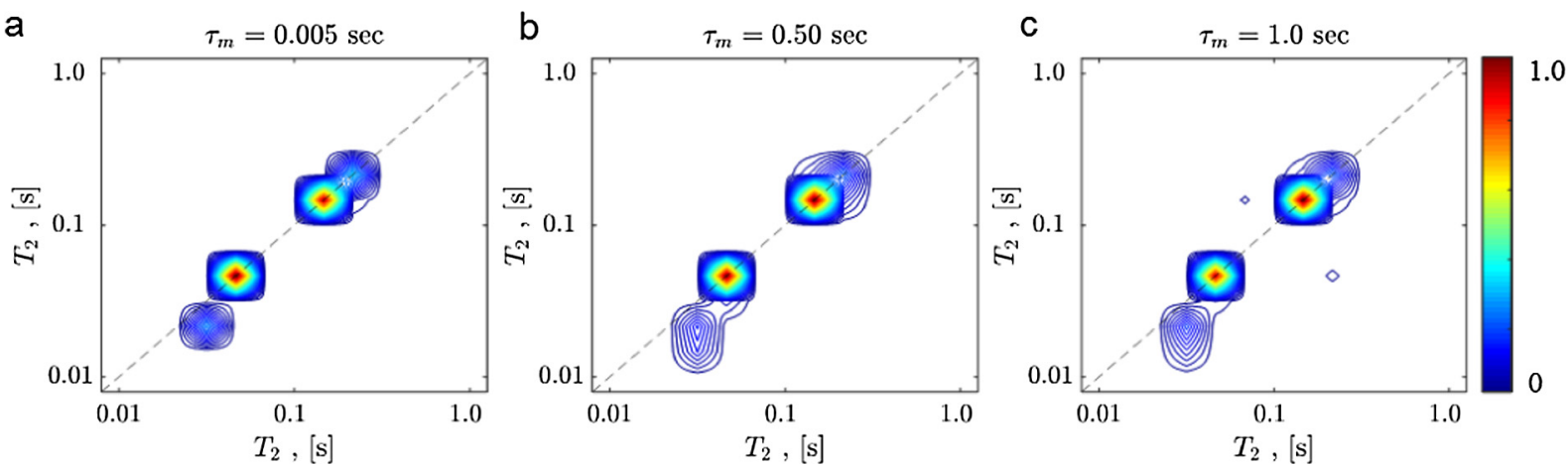

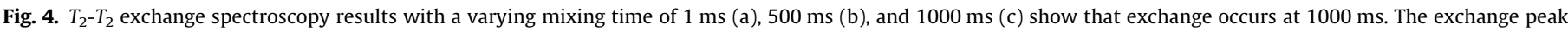
intensity at $1000 \mathrm{~ms}$ is about $1.5 \%$ of the total signal respectively. 
Table 1

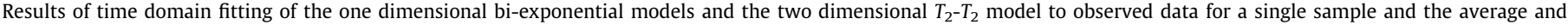
standard deviations of the results for the three samples used in this study.

\begin{tabular}{|c|c|c|c|c|}
\hline & Representative sample 1 -D results & Representative sample 2-D results & Average 1 -D results & Average $2-D$ results \\
\hline$T_{1 \mathrm{~A}}[\mathrm{~ms}]$ & 2300 & 1500 & $2167 \pm 94$ & $3493 \pm 793$ \\
\hline$T_{1 \mathrm{~B}}[\mathrm{~ms}]$ & 2300 & 1000 & $2167 \pm 94$ & $2404 \pm 673$ \\
\hline$T_{2 \mathrm{~A}}[\mathrm{~ms}]$ & 150 & 180 & $182 \pm 28$ & $224 \pm 48$ \\
\hline$T_{2 \mathrm{~B}}[\mathrm{~ms}]$ & 37 & 33 & $77 \pm 2$ & $53.7 \pm 27.9$ \\
\hline$M_{\mathrm{A}}[\%]$ & 49 & 46 & $36 \pm 9.2$ & $35.6 \pm 10.3$ \\
\hline$M_{\mathrm{B}}[\%]$ & 51 & 54 & $64 \pm 9.2$ & $64.3 \pm 10.3$ \\
\hline$k_{a}^{-1}[\mathrm{~s}]$ & - & 1.33 & - & $1.56 \pm 0.2$ \\
\hline
\end{tabular}

of mixing between the sites is such that the two sites display a single, averaged relaxation time that is extracted by the $1 \mathrm{D}$ fits and clearly suggested by the $2 \mathrm{D}$ results. In the case of the $2 \mathrm{D}$ results, the introduction of the parameter $k_{a}$ allows some separation of the $T_{1}$ values in the two sites for individual samples. However, that separation is dominated by the constraints on the fitting routine. Since the routine is fitting one number, the apparent $T_{1}$, with two values, $T_{1 \mathrm{~A}}$ and $T_{1 \mathrm{~B}}$, there are too many degrees of freedom to accurately and precisely fit the value. This is reflected in the large standard deviations in the fitting of $T_{1 \mathrm{~A}}$ and $T_{1 \mathrm{~B}}$. On the other hand, the time scale for transverse relaxation is an order of magnitude shorter than the time scale for exchange and so there is little to no mixing effect on $T_{2}$. The $2 \mathrm{D}$ results thus provide two distinct, not exchange weighted values for $T_{2}$ and, essentially, those same values are also found by the 1D $T_{2}$ fitting process.

Insight into the cartilage physical structure and the data-fitting process is obtained by comparing the probability distributions calculated from the ILT of the data and model fit predictions. In Figs. 5 and 6, ILT's of the data, ILT's of the time domain model predictions, and the analytical model results of the time domain model predictions are presented for a single sample representative of all the results. In each (a) is an ILT of the data from the experiment, (b) is the ILT of the predictions of the 2D time domain model using the best-fit parameter values and (c) is the analytical model that is predicted by the elements of the peak matrix, $\boldsymbol{P}$, for the sequence when the fit parameters are entered into Eq. (4) for $T_{2}-T_{2}$ and Eq. (5) for $T_{1}-T_{2}$. To perform this analysis, the raw time domain data was first fit to Eq (3). The extracted values were then used to create the noise-free model. ILT analysis of the time domain data (a) and the noise free model (b) are compared to the predicted ILT results (c).
Comparison of ILT predictions for the $T_{1}-T_{2}$ sequence (Fig. 5) shows similar results for the data and the fitted model. Two sites are present in both plots and while two $T_{2}$ values are identified, only one $T_{1}$ value is observed. The ILT predicted by the elements of the analytical model matrix (Fig. 5c) exhibits four peaks of different amplitude corresponding to the eigenvalues of the solution matrix. These peaks correspond to two "on diagonal" peaks and two exchange peaks. The on diagonal peaks represent intrinsic and exchange weighted intrinsic relaxation and are the peaks with the shortest $T_{1}, T_{2}$ and longest $T_{1}, T_{2}$ bottom left and top right respectively. The other two peaks are exchange peaks, the long $T_{1}$ associated with the short $T_{2}$ and the short $T_{1}$ associated with the long $T_{2}$. The results of the analytical model clearly show two primary peaks: one located by the longest $T_{1}$ and $T_{2}$ values and the second, exchange peak located by the long $T_{1}$ and the short $T_{2}$ values. These peaks are consistent with the $1 \mathrm{D}$ results (Fig. 2) and the ILT of the $T_{1}-T_{2}$ data in percent signal and $T_{1}$ and $T_{2}$ value (Fig. 3a, Fig. 5a). The presence of the exchange peak indicates that mixing between populations of spins is occurring on the time scale of longitudinal relaxation in the system [43-46]. Note that the amplitude of the off-diagonal peak below the diagonal is predicted to be negative, indicated by the square in Fig. 5c. In this example, the amplitude of the negativeamplitude peak is below $1 \%$ of the signal and therefore has little impact on the ILT. The results of Eq. (4) (Fig. 5c) show agreement with the ILT of $T_{1}-T_{2}$ data (Fig. 5a) and model predictions (Fig. 5b). All three data visualization methods show two populations with a single $T_{1}$ and distinct $T_{2}$ values, which is evidence for exchange weighting of measured $T_{1}$ relaxation in the $T_{1}-T_{2}$ correlation experiment.
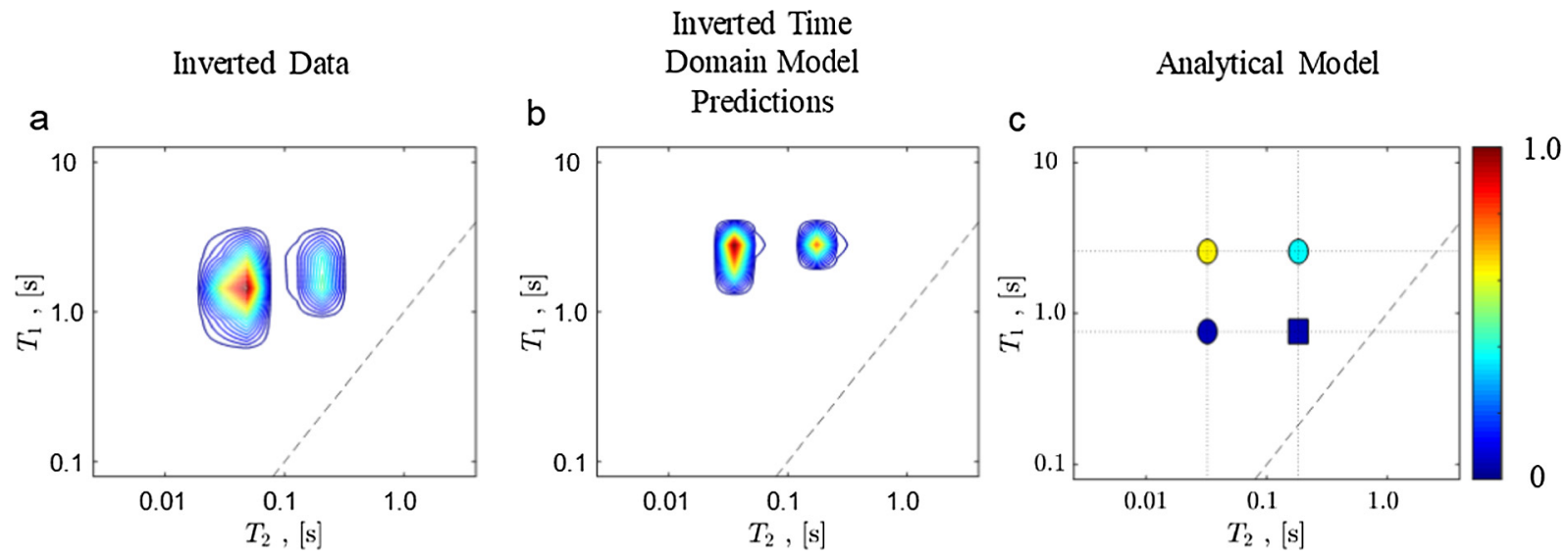

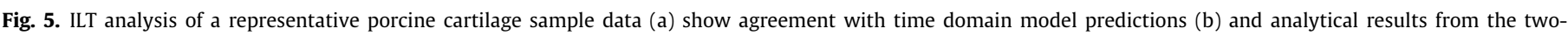

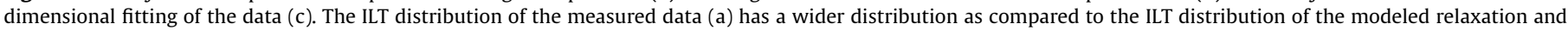

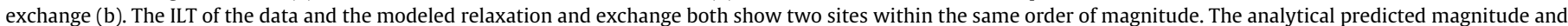

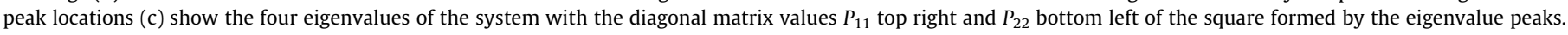

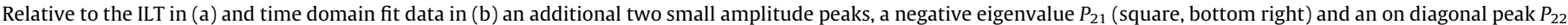

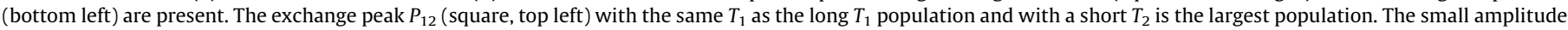
on-diagonal peak $P_{22}$ is not resolved in the ILT of the data. 

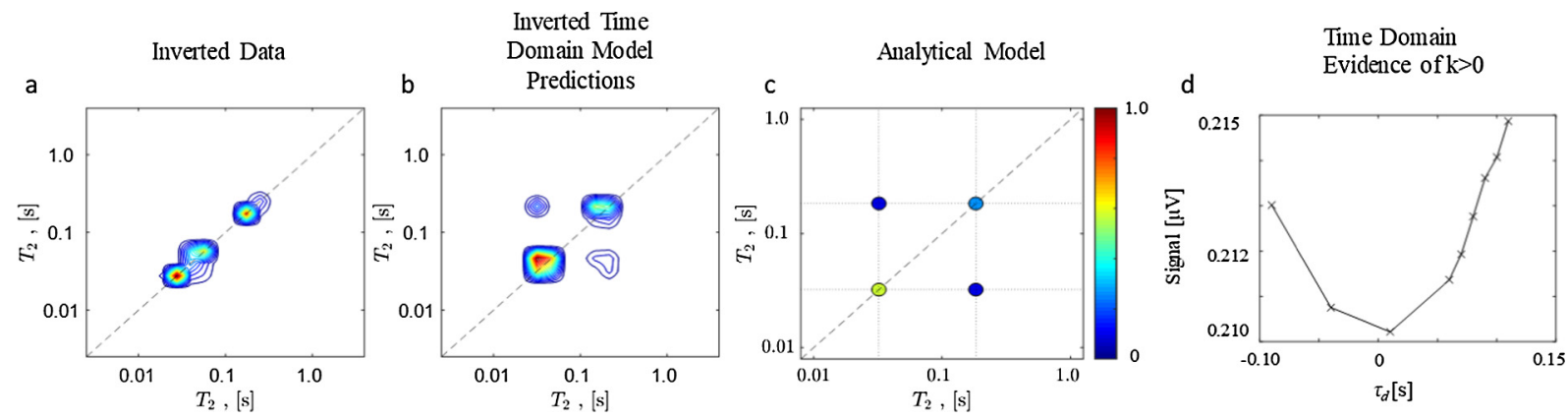

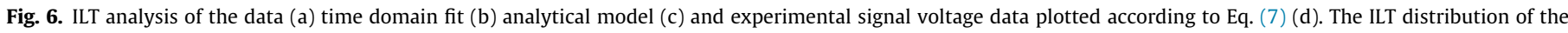

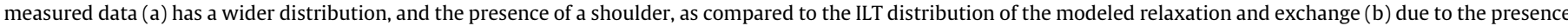

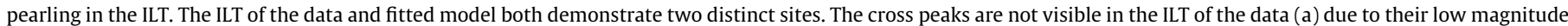

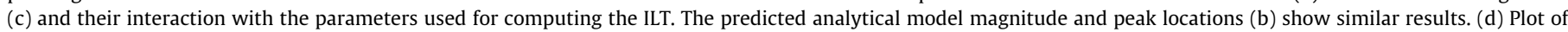

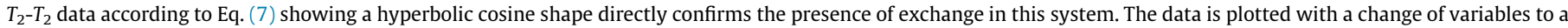
$\tau_{d}$ projection at $\tau_{\mathrm{s}}=0.110 \mathrm{~s}$.

A comparison of the ILTs for the $T_{2}-T_{2}$ measurement at $500 \mathrm{~ms}$ is presented in Fig. 6. The exchange peaks in the ILT of the data are small in amplitude and quite close to the noise level in the computed result, and hence not resolved (Fig. 6a). The exchange peaks are more clearly visible in the ILT of the time domain model prediction (Fig. 6b) and the analytical solution (Fig. 6c), where it is evident that their amplitudes are less than $10 \%$ of the total signal. There is reasonable agreement on the location and amplitude of peaks on the diagonal across all three analyses of this data. The data and analysis demonstrate a limitation of the ILT for resolving small peaks in the $T_{2}-T_{2}$ exchange experiments. Additionally, as a direct demonstration of the existence of diffusive exchange in the system, the voltage signal acquired at $\tau_{\mathrm{s}}=0.110 \mathrm{~s}$ is plotted as a function of $\tau_{\mathrm{d}}$ and is shown to exhibit curvature due to the hyperbolic cosine modulation of the signal according to Eq. (7).

In the porcine cartilage studied here, two $T_{2}$ populations correlated to a restricted diffusion coefficient of about $75 \%$ of water (Fig. 4), a value consistent with restricted water in cartilage [47] is observed. This finding is consistent with previous studies which have attributed the multiple $T_{2}$ populations to the environments of fluid in a pore and fluid interacting with collagen $[8,10]$. In contrast, the single observed $T_{1}$ relaxation rate is impacted by exchange and hence does not represent an intrinsic relaxation rate. The appearance of a single $T_{1}$ indicates that averaging of $T_{1}$ has occurred because the exchange time is within a decade of the observed $T_{1}$. Since exchange is on the order of $T_{1}$, the intrinsic $T_{1}$ relaxation of this system was not directly measured (Fig. 5a). This is confirmed as an exchange peak in the $T_{1}-T_{2}$ correlation of the analytical model. The peak in the $T_{1}-T_{2}$ map at $T_{2} \sim 0.07 \mathrm{~s}$ is an artifact of exchange. Time domain analysis is not capable of finding the intrinsic $T_{1}$ relaxation, however fitted values for intrinsic relaxation rates and $T_{1}-T_{2}$ correlations (Fig. $5 \mathrm{c}$ ) provide insight into the relaxation processes and reasonable quantitative estimates of the intrinsic $T_{1}$ values.

The time scale of the measured exchange, $k_{a}^{-1}$, is a physical exchange mediated by diffusive motions, based on it's magnitude of $1 \mathrm{~s}$. Observations of $T_{2}$ averaging in cartilage with a change in solvent have been attributed to chemical exchange [12] due to proton exchange in an aqueous phosphate buffer being faster than proton exchange in water and give a timescale on the order of $\sim 10^{-9} \mathrm{~s}$ [48]. The time scale measured here is $1.54 \mathrm{~s}$, a value too large for chemical exchange processes. Using the average diffusion coefficient from the $D-T_{2}$ correlations (Fig. 2c), the diffusive length, $l$, of fluid molecules based on the exchange rate timescale can be determined using the Einstein relation [32], $l=\sqrt{6 D k^{-1}}$. An average diffusive length of $140 \pm 50 \mu \mathrm{m}$ is predicted for the material studied here. This diffusive length is in agreement with distance between spatially varying $T_{2}$ relaxation rates observed in healthy canine articular cartilage tissues [6]. Specifically, a long-lived $T_{2}$ population exists near the surface and a short $T_{2}$ population exists in the middle zone and deep zone of the tissue. The $T_{2}$ gradually changes as a function of distance between the minimum and maximum due to changing fiber orientations relative to the applied $B_{0}$ which generate a magic angle effect on the measured relaxation. Additionally, the apparent $T_{2}$ values in this study agree with a study of healthy porcine cartilage oriented with the applied magnetic field and at the magic angle. The observation of two $T_{2}$ populations occurs when the sample is oriented with the magnetic field, but only one population is observed when the sample is oriented at the magic angle [49]. In this study, the cartilage samples, and therefore collagen fiber orientations, were oriented randomly and showed a $T_{2}$ distribution similar to the $T_{2}$ distribution measured for porcine cartilage aligned between 0 and $30^{\circ}$ with the magnetic field [49]. This is shown by the width of the ILT distributions in $T_{2}$ (Fig. 2a). Thus, the physical, diffusion-mediated exchange observed is due to the translational diffusion of water between regions of cartilage fibril orientation relative to the magnetic field that are a distance of the order of $100 \mu \mathrm{m}$ apart.

The direct identification of physical exchange in an avascular tissue such as cartilage is relevant to understanding diffusion of fluid and nutrients from the bone and synovial fluid, that are essential for cell survival and tissue function [50]. It has also been hypothesized that fluid in different environments in cartilage, such as that associated with macromolecules and in the pore space of cartilage, contribute differently to the response to loading [51]. Data on diffusive exchange and coupling between these environments, such as that presented here can provide new information about the function of fluid in this complex material.

\section{Conclusions}

In this paper time domain fitting and analytical model solutions combined with 2D relaxation-time correlation and exchange experiments are shown to provide detailed characterization of diffusive exchange in porcine cartilage. Measurement of the relaxation behavior of cartilage using $T_{1}-T_{2}$ correlation experiments indicates two populations that communicate via diffusion mediated exchange. The exchange process averages the $T_{1}$ relaxation processes. Hence intrinsic $T_{1}$ values are difficult to extract and a single $T_{1}$ value is observed. However, it is still possible to provide intrinsic $T_{2}$ values using a two-site model. Applying time domain analysis and analytical modeling, the presence of diffusive exchange averaging of the measured $T_{1}$ is demonstrated. To further analyze the diffusive exchange process $T_{2}-T_{2}$ experiments are used 
and it is demonstrated that only a single mixing time is required to determine the exchange timescale, when combined with time domain fitting and the analytical solution. The intrinsic $T_{2}$ values, percentage populations, and an exchange time are determined with these methods. The exchange timescale from the $T_{2}-T_{2}$ exchange experiment is of the same order of magnitude $\sim 1 \mathrm{~s}$, as that determined from the analytical model of the $T_{1}-T_{2}$ correlation experiment. $D-T_{2}$ correlation experiments provide the translational diffusivity of the two observed $T_{2}$ populations and indicate they are water associated with the cartilage matrix, not free water. Using the measured diffusion and the exchange time determined from the relaxation experiment, a length scale of $\sim 100 \mu \mathrm{m}$ consistent with diffusion between oriented fiber domains of different $T_{2}$ is calculated.

\section{Acknowledgments}

Funding for SEM was provided by the US National Science Foundation East Asia Pacific Summer Internship Program (1614189), JEM was funded by Bucknell University Sabbatical Leave funds. FZ was funded by the New Zealand Ministry for Business, Innovation and Employment. This work is funded in part by a US National Science Foundation (1554708) award to RKJ. The authors thank B. Blümich and M. Van Landeghem for sharing code from Ref. [16].

\section{References}

[1] Y. Xia, T. Farquhar, N. Burton-Wurster, E. Ray, L.W. Jelinski, Diffusion and relaxation mapping of cartilage-bone plugs and excised disks using microscopic magnetic resonance imaging, Magn. Reson. Med. 31 (3) (1994) 273-282.

[2] Y. Xia, Relaxation anisotropy in cartilage by NMR microscopy (muMRI) at 14 microm resolution, Magn. Reson. Med. 39 (6) (1998) 941-949.

[3] Y. Xia, Heterogeneity of cartilage laminae in MR imaging, J. Magn. Reson. Imag. 11 (6) (2000) 686-693.

[4] Y. Xia, J.B. Moody, H. Alhadlaq, Orientational dependence of T2 relaxation in articular cartilage: a microscopic MRI (microMRI) study, Magn. Reson. Med. 48 (3) (2002) 460-469, https://doi.org/10.1002/mrm.10216.

[5] H.A. Alhadlaq, Y. Xia, J.B. Moody, J.R. Matyas, Detecting structural changes in early experimental osteoarthritis of tibial cartilage by microscopic magnetic resonance imaging and polarised light microscopy, Ann. Rheum. Dis. 63 (6) (2004) 709-717, https://doi.org/10.1136/ard.2003.011783.

[6] S. Zheng, Y. Xia, Multi-components of T2 relaxation in ex vivo cartilage and tendon, J. Magn. Reson. 198 (2) (2009) 188-196, https://doi.org/10.1016/j. jmr.2009.02.005.

[7] K.R. Brownstein, C.E. Tarr, Importance of classical diffusion in NMR studies of water in biological cells, Phys. Rev. A 19 (6) (1979) 2446-2453.

[8] D.A. Reiter, P.C. Lin, K.W. Fishbein, R.G. Spencer, Multicomponent T2 relaxation analysis in cartilage, Magn. Reson. Med. 61 (4)(2009) 803-809, https://doi.org/ $10.1002 / \mathrm{mrm} 21926$.

[9] D.A. Reiter, R.A. Roque, P.-C. Lin, S.B. Doty, N. Pleshko, R.G. Spencer, Improved specificity of cartilage matrix evaluation using multiexponential transverse relaxation analysis applied to pathomimetically degraded cartilage, NMR Biomed. 24 (10) (2011) 1286-1294, https://doi.org/10.1002/nbm.1690.

[10] D.A. Reiter, R.L. Magin, W. Li, J.J. Trujillo, M. Pilar Velasco, R.G. Spencer, Anomalous T2 relaxation in normal and degraded cartilage, Magn. Reson. Med. 76 (3) (2016) 953-962, https://doi.org/10.1002/mrm.25913.

[11] N. Wang, Y. Xia, Experimental issues in the measurement of multi-component relaxation times in articular cartilage by microscopic MRI, J. Magn. Reson. 235 (2013) 15-25, https://doi.org/10.1016/j.jmr.2013.07.001.

[12] S. Zheng, Y. Xia, Effect of phosphate electrolyte buffer on the dynamics of water in tendon and cartilage, NMR Biomed. 22 (2) (2009) 158-164, https:// doi.org/10.1002/nbm.1294.

[13] S. Zheng, Y. Xia, The collagen fibril structure in the superficial zone of articular cartilage by $\mu$ MRI, Osteoarthr. Cartilage 17 (11) (2009) 1519-1528, https://doi. org/10.1016/j.joca.2009.05.013.

[14] S. Zheng, Y. Xia, On the measurement of multi-component T2 relaxation in cartilage by MR spectroscopy and imaging, Magn. Reson. Imag. 28 (4) (2010) 537-545, https://doi.org/10.1016/j.mri.2009.12.006.

[15] J.H. Lee, C. Labadie, C.S. Springer, G.S. Harbison, Two-dimensional inverse Laplace transform NMR: altered relaxation times allow detection of exchange correlation, J. Am. Chem. Soc. 115 (17) (1993) 7761-7764, https://doi.org/ 10.1021/ja00070a022.

[16] M. Van Landeghem, A. Haber, J.-B. D’Espinose De Lacaillerie, B. Blümich, Analysis of multisite 2D relaxation exchange NMR, Concepts Magn. Reson. Part A 36A (3) (2010) 153-169, https://doi.org/10.1002/cmr.a.20157.
[17] R.D. Dortch, R.A. Horch, M.D. Does, Development, simulation, and validation of NMR relaxation-based exchange measurements, J. Chem. Phys. 131 (16) (2009) 164502, https://doi.org/10.1063/1.3245866.

[18] P.J. McDonald, J.P. Korb, J. Mitchell, L. Monteilhet, Surface relaxation and chemical exchange in hydrating cement pastes: a two-dimensional NMR relaxation study, Phys. Rev. E 72 (1) (2005) 011409.

[19] B. Hills, S. Benamira, N. Marigheto, K. Wright, T1-T2 correlation analysis of complex foods, Appl. Magn. Reson. 26 (4) (2004) 543-560, https://doi.org/ $10.1007 /$ bf03166582.

[20] J. Warner, S. Donell, K. Wright, L. Venturi, B. Hills, The characterisation of mammalian tissue with 2D relaxation methods, Magn. Reson. Imag. 28 (7) (2010) 971-981, https://doi.org/10.1016/j.mri.2010.03.015.

[21] K.E. Washburn, P.T. Callaghan, Tracking pore to pore exchange using relaxation exchange spectroscopy, Phys. Rev. Lett. 97 (17) (2006) 175502.

[22] R. Bai, D. Benjamini, J. Cheng, P.J. Basser, Fast, accurate 2D-MR relaxation exchange spectroscopy (REXSY): Beyond compressed sensing, J. Chem. Phys. 145 (15) (2016) 154202, https://doi.org/10.1063/1.4964144.

[23] S. Ahola, V.V. Zhivonitko, O. Mankinen, G. Zhang, A.M. Kantola, H.-Y. Chen, C. Hilty, I.V. Koptyug, V.V. Telkki, Ultrafast multidimensional Laplace NMR for a rapid and sensitive chemical analysis, Nat. Commun. 6 (2015), https://doi.org/ $10.1038 /$ ncomms9363.

[24] R.M. Henkelman, G.J. Stanisz, J.K. Kim, M.J. Bronskill, Anisotropy of NMR properties of tissues, Magn. Reson. Med. 32 (5) (1994) 592-601, https://doi. org/10.1002/mrm.1910320508.

[25] D.A. Reiter, A. Peacock, R.G. Spencer, Effects of frozen storage and sample temperature on water compartmentation and multiexponential transverse relaxation in cartilage, Magn. Reson. Imag. 29 (4) (2011) 561-567, https://doi. org/10.1016/j.mri.2010.10.011.

[26] Y. Xia, S. Zheng, A. Bidthanapally, Depth-dependent profiles of glycosaminoglycans in articular cartilage by microMRI and histochemistry, J. Magn. Reson. Imag. 28 (1) (2008) 151-157, https://doi.org/10.1002/ jmri.21392.

[27] N. Wang, Y. Xia, Depth and orientational dependencies of MRI T2 and T1 $\rho$ sensitivities towards trypsin degradation and Gd-DTPA2 - presence in articular cartilage at microscopic resolution, Magn. Reson. Imag. 30 (3) (2012) 361-370, https://doi.org/10.1016/j.mri.2011.10.004.

[28] Y. Xia, S. Zheng, M. Szarko, J. Lee, Anisotropic properties of bovine nasal cartilage, Microsc. Res. Tech. 75 (3) (2012) 300-306, https://doi.org/ 10.1002/jemt.21058.

[29] L. Venkataramanan, S. Yi-Qiao, M.D. Hurlimann, Solving Fredholm integrals of the first kind with tensor product structure in 2 and 2.5 dimensions, IEEE Trans. Signal Process. 50 (5) (2002) 1017-1026, https://doi.org/10.1109/ 78.995059.

[30] Y.Q. Song, L. Venkataramanan, M.D. Hürlimann, M. Flaum, P. Frulla, C. Straley, T1-T2 correlation spectra obtained using a fast two-dimensional laplace inversion, J. Magn. Reson. 154 (2) (2002) 261-268, https://doi.org/10.1006/ jmre.2001.2474.

[31] L.M. Schwartz, D.L. Johnson, J. Mitchell, T.C. Chandrasekera, E.J. Fordham, Modeling two-dimensional magnetic resonance measurements in coupled pore systems, Phys. Rev. E 88 (3) (2013) 032813.

[32] L. Monteilhet, J.P. Korb, J. Mitchell, P.J. McDonald, Observation of exchange of micropore water in cement pastes by two-dimensional T2 nuclear magnetic resonance relaxometry, Phys. Rev. E 74 (6) (2006) 061404.

[33] J. Mitchell, J.D. Griffith, J.H.P. Collins, A.J. Sederman, L.F. Gladden, M.L. Johns, Validation of NMR relaxation exchange time measurements in porous media, J. Chem. Phys. 127 (23) (2007) 234701, https://doi.org/10.1063/1.2806178.

[34] H.M. McConnell, Reaction rates by nuclear magnetic resonance, J. Chem. Phys. 28 (3) (1958) 430-431, https://doi.org/10.1063/1.1744152.

[35] R. Song, Y.Q. Song, M. Vembusubramanian, J.L. Paulsen, The robust identification of exchange from T2-T2 time-domain features, J. Magn. Reson. 265 (2016) 164-171, https://doi.org/10.1016/j.jmr.2016.02.001.

[36] S. Meiboom, D. Gill, Modified spin-echo method for measuring nuclear relaxation times, Rev. Sci. Instrum. 29 (8) (1958) 688-691, https://doi.org/ 10.1063/1.1716296.

[37] M.D. Hürlimann, L. Venkataramanan, Quantitative measurement of twodimensional distribution functions of diffusion and relaxation in grossly inhomogeneous fields, J. Magn. Reson. 157 (1) (2002) 31-42, https://doi.org/ 10.1006/jmre.2002.2567.

[38] Y. Qiao, P. Galvosas, P.T. Callaghan, Diffusion correlation NMR spectroscopic study of anisotropic diffusion of water in plant tissues, Biophys. J . 89 (4) (2005) 2899-2905, https://doi.org/10.1529/biophysj.105.064709.

[39] J.S. Alper, R.I. Gelb, Standard errors and confidence intervals in nonlinear regression: comparison of Monte Carlo and parametric statistics, J. Phys. Chem. 94 (11) (1990) 4747-4751, https://doi.org/10.1021/j100374a068.

[40] S.E. Mailhiot, N.H. Williamson, J.R. Brown, J.D. Seymour, S.L. Codd, R.K. June $\mathrm{T}_{1}-\mathrm{T}_{2}$ correlation and biopolymer diffusion within human osteoarthritic cartilage measured with nuclear magnetic resonance, Appl. Magn. Reson. 48 (4) (2017) 407-422, https://doi.org/10.1007/s00723-017-0869-x.

[41] P. Galvosas, Y. Qiao, M. Schönhoff, P.T. Callaghan, On the use of 2D correlation and exchange NMR spectroscopy in organic porous materials, Magn. Reson. Imag. 25 (4) (2007) 497-500, https://doi.org/10.1016/j.mri.2006.11.009.

[42] K.E. Washburn, P.T. Callaghan, Propagator resolved transverse relaxation exchange spectroscopy, J. Magn. Reson. 186 (2) (2007) 337-340, https://doi. org/10.1016/j.jmr.2007.02.011. 
[43] S. Rodts, D. Bytchenkoff, Structural properties of 2D NMR relaxation spectra of diffusive systems, J. Magn. Reson. 205 (2) (2010) 315-318, https://doi.org/ 10.1016/j.jmr.2010.04.021.

[44] D. Bytchenkoff, S. Rodts, Structure of the two-dimensional relaxation spectra seen within the eigenmode perturbation theory and the two-site exchange model, J. Magn. Reson. 208 (1) (2011) 4-19, https://doi.org/10.1016/j. jmr.2010.09.007.

[45] Y.Q. Song, L. Zielinski, S. Ryu, Two-dimensional NMR of diffusion systems, Phys. Rev. Lett. 100 (24) (2008) 248002, https://doi.org/10.1103/PhysRevLett. 100.248002 .

[46] Y.Q. Song, G. Carneiro, L.M. Schwartz, D.L. Johnson, Experimental identification of diffusive coupling using 2D NMR, Phys, Rev. Lett. 113 (23) (2014) 235503.

[47] Y. Xia, T. Farquhar, N. Burton-Wurster, M. Vernier-Singer, G. Lust, L.W. Jelinski, Self-diffusion monitors degraded cartilage, Arch. Biochem. Biophys. 323 (2) (1995) 323-328, https://doi.org/10.1006/abbi.1995.9958.
48] Z. Luz, S. Meiboom, Rate and mechanism of proton exchange in aqueous solutions of phosphate buffer, J. Am. Chem. Soc. 86 (22) (1964) 4764-4766, https://doi.org/10.1021/ja01076a006.

[49] W. Gründer, M. Wagner, A. Werner, MR-microscopic visualization of anisotropic internal cartilage structures using the magic angle technique, Magn. Reson. Med. 39 (3) (1998) 376-382, https://doi.org/10.1002/ mrm.1910390307.

[50] A.J. Sophia Fox, A. Bedi, S.A. Rodeo, The basic science of articular cartilage: structure, composition, and function, Sports Health. 1 (6) (2009) 461-468, https://doi.org/10.1177/1941738109350438.

[51] V.C. Mow, S.C. Kuei, W.M. Lai, C.G. Armstrong, Biphasic creep and stress relaxation of articular cartilage in compression? Theory and experiments, J. Biomech. Eng. 102 (1) (1980) 73-84. 